\title{
Concurrent Extramedullary Hematopoiesis and Pseudometastatic Hypervascular Lesions Masquerading as Metastases in Liver in a Case of Neuroblastoma
}

\author{
Abhijeet Taori ${ }^{1}$ Nazih Shenouda ${ }^{1}$ Dina El Demellawy ${ }^{2}$ Rita Putnins ${ }^{1}$ \\ ${ }^{1}$ Department of Medical Imaging, CHEO, University of Ottawa, \\ Ontario, Canada \\ 2 Department of Pathology, CHEO, University of Ottawa, Ontario, \\ Canada \\ Address for correspondence Abhijeet Taori, MD, DNB, EDiR, \\ Department of Radiology, CHEO, 401 Smyth Road, Ottawa, ON K1H \\ 8L1, Canada (e-mail: abhijittaori@gmail.com). \\ Indian J Radiol Imaging 2021;31:714-718.
}

\begin{abstract}
Keywords

- extramedullary hematopoiesis

- neuroblastoma

- pseudometastatic hypervascular lesions

Neuroblastoma is a common embryonic tumor presenting in childhood. Improving treatment protocols which include a combination of chemotherapy, surgical resection, hematopoietic stem cell rescue, and radiation therapy have tremendously improved outcomes. Childhood survivors are at risk of developing lesions which may mimic metastases. It is essential to accurately diagnose these due to its prognostic implications.
\end{abstract}

\section{Introduction}

Neuroblastoma is a malignant tumor of the sympathetic chain involving the primitive neural crest cells. Neuroblastoma often presents with metastases at diagnosis. The commonest locations include bone, lymph nodes, liver, and soft tissues. $^{1}$

Extramedullary hematopoiesis (EMH) usually occurs in the setting of hematological disease $\mathrm{e}^{2}$ but rarely develops in cases of malignant solid tumors. ${ }^{3}$ EMH in these patients might easily be misdiagnosed as metastasis. ${ }^{4}$

Pseudometastatic hypervascular lesions of the liver may be detected in children previously treated for malignant tumor. The proposed hypothesis is that they are benign regenerative lesions secondary to treatment, most commonly postchemotherapy or stem cell transplant. ${ }^{5}$

EMH and pseudometastatic hypervascular lesions of the liver are nonaggressive and have a benign course. We have not come across any report of EMH in liver in a case of neuroblastoma. Additionally, we also found there were multiple pseudometastatic hypervascular lesions in the liver in our case; thus, this case represents a rare and a unique presentation.

\section{Case Report}

A 21-month-old girl initially presented to us with a 2-week history of ataxia, loss of consciousness, bruises on forehead, nystagmus, tremor, mydriasis, and morning irritability. She was referred to radiology by the emergency physician to exclude a posterior fossa tumor. Magnetic resonance imaging (MRI) of the brain and spine was within normal limits. However, a large $3 \times 2.5 \mathrm{~cm}$ suprarenal mass was incidentally discovered on the images of the thoracolumbar spine.

The findings on subsequent ultrasound (US) and MRI of the abdomen were consistent with a neuroblastoma
DOI https://doi.org/ 10.1055/s-0041-1736400 ISSN 0971-3026.

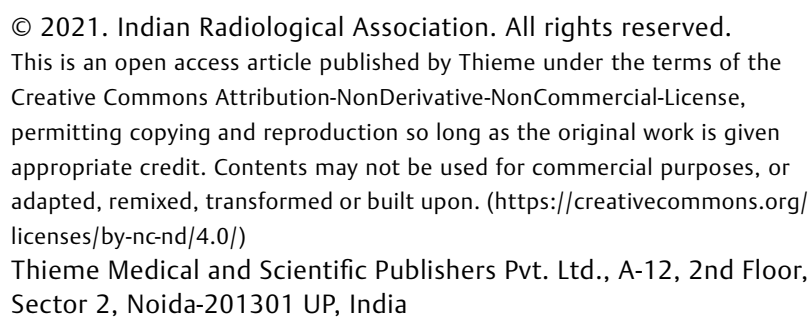




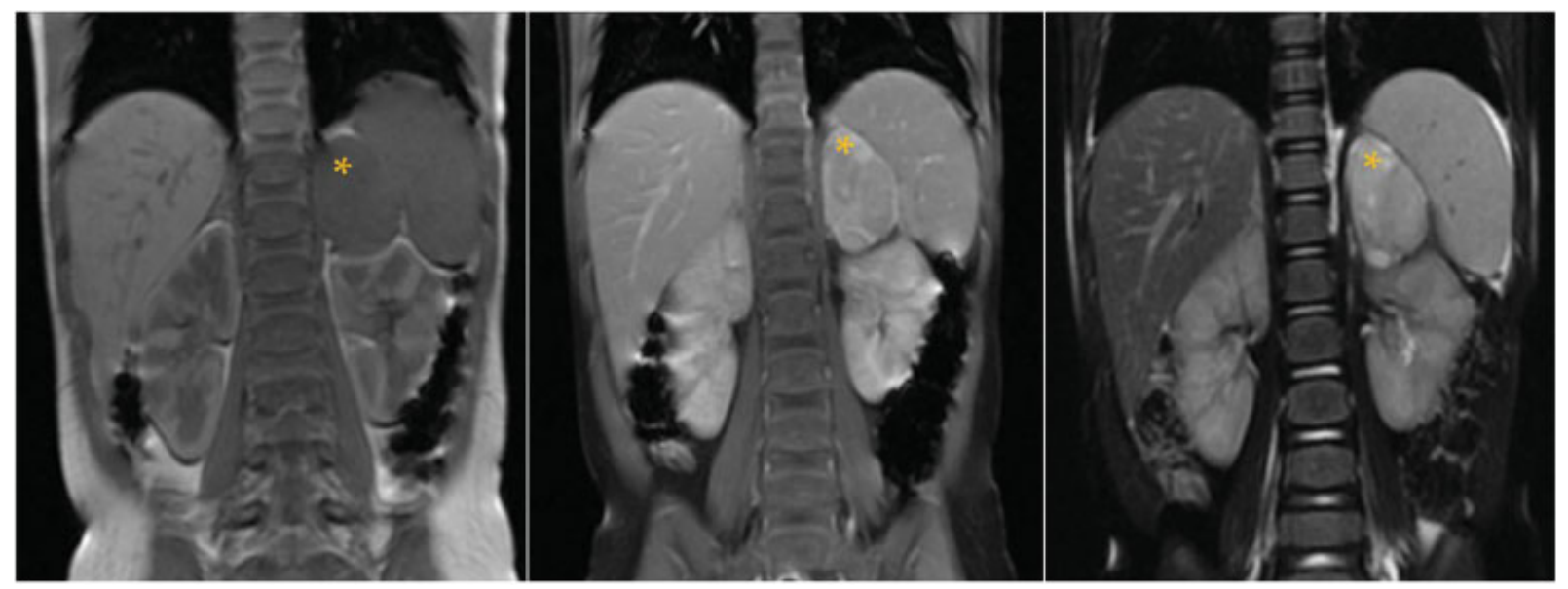

Fig. 1 Magnetic resonance imaging of the abdomen coronal T1, T1c +, and T2 images: A large left suprarenal lobulated lesion with heterogeneous postcontrast enhancement and mass effect on the adjacent spleen/kidney without invasion. No extension into the adjacent neural foramina. Note that visualized liver appears normal.

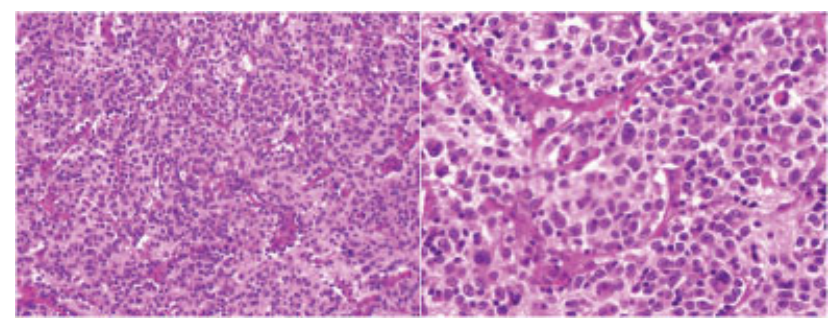

Fig. 2 Medium- and high-power microscopic views: There are small, round blue cells with scant cytoplasm arranged in lobules separated by thin fibrovascular septa and embedded in abundant pink fibrillary matrix/neutrophils; salt and pepper chromatin that is characteristic of neuroblastoma cells (HPS $\times 200$ and $\times 400)$.

(-Fig. 1). At the time of diagnosis, the tumor was localized and showed no evidence of local or regional invasion, as defined by the image-defined risk factors. The tumor represented a Stage 1 neuroblastoma with opsoclonusmyoclonus-ataxia syndrome.

A left adrenalectomy was performed and pathology confirmed the neuroblastoma (-Fig. 2). Our follow-up MRI protocol comprised coronal T1, T2FS, axial 3D T1 (with in/out phase), T2 images, and three plane postcontrast with gadolinium. Diffusion was acquired in axial plane. Postsurgery MRI of the abdomen demonstrated no residual soft tissue tumor. This was followed by intravenous immunoglobulin (IVIG), prednisone, and cyclophosphamide monthly to treat the opsoclonus-myoclonus-ataxia syndrome. At a 3-month follow-up, no tumor recurrence was detected at the surgical bed. However, a single very small T1 iso/hypointense and T2 hyperintense lesion with equivocal enhancement was discovered within the liver in segment $6 / 5$, which was thought to be metastases versus hemangioma (-Fig. 3). There was no signal abnormality diffusion-weighted images or signal dropout on opposed phase images. A corroborative abdominal US could not identify this lesion.

A follow-up MRI in 3 months showed interval growth of this lesion with avid enhancement but no specific washout characteristics. Due to interval growth (-Fig. 3), the possibility of metastases was thought to be more likely; hence, a biopsy was recommended. The biopsy of this liver lesion was attempted on two separate occasions, without definitive diagnosis.

The liver lesion had increased in size over 3 months and the opsoclonus-myoclonus had worsened. Our patient therefore underwent further chemotherapy to see whether the size of the lesion would decrease. It was felt that chemotherapy would have less morbidity than surgical resection of the liver lesion. During this time, she continued to receive IVIG and steroids for the opsoclonus-myoclonus without success. A stem cell collection was performed in preparation for high-dose chemotherapy with autologous stem cell rescue.

After the completion of two cycles of chemotherapy with bone marrow transplant, the MRI showed multiple similar larger lesions scattered within the liver parenchyma. Another MRI after 3 months showed further increase in number of lesions within the liver (-Fig. 4). At tumor board, it was decided to pursue open biopsy for diagnosis. The pathology on the peripheral wedge biopsy of liver surprisingly showed multifocal EMH with dilated sinusoids. There was no evidence of neoplasia. The same diagnosis was confirmed after consultation with pathology at a second tertiary pediatric hospital (-Fig. 5). Nevertheless, due to high risk of metastases in liver in cases of neuroblastoma, the lesions were still followed up every 3 months. Subsequent MRI studies over 2 years showed continued increase in size and number of the lesions in the liver.

A repeat biopsy was done. The pathology showed startling results. A multicenter pediatric hospital review deemed them to be pseudometastatic hypervascular lesions along with EMH (-Fig. 6). On follow-up MRI examinations, these lesions continued to increase in size, eventually showing stability. The patient now continues to have MRI examinations every 6 months (-Fig. 7).

The final diagnosis in this case was neuroblastoma presenting as opsoclonus-myoclonus syndrome with later 


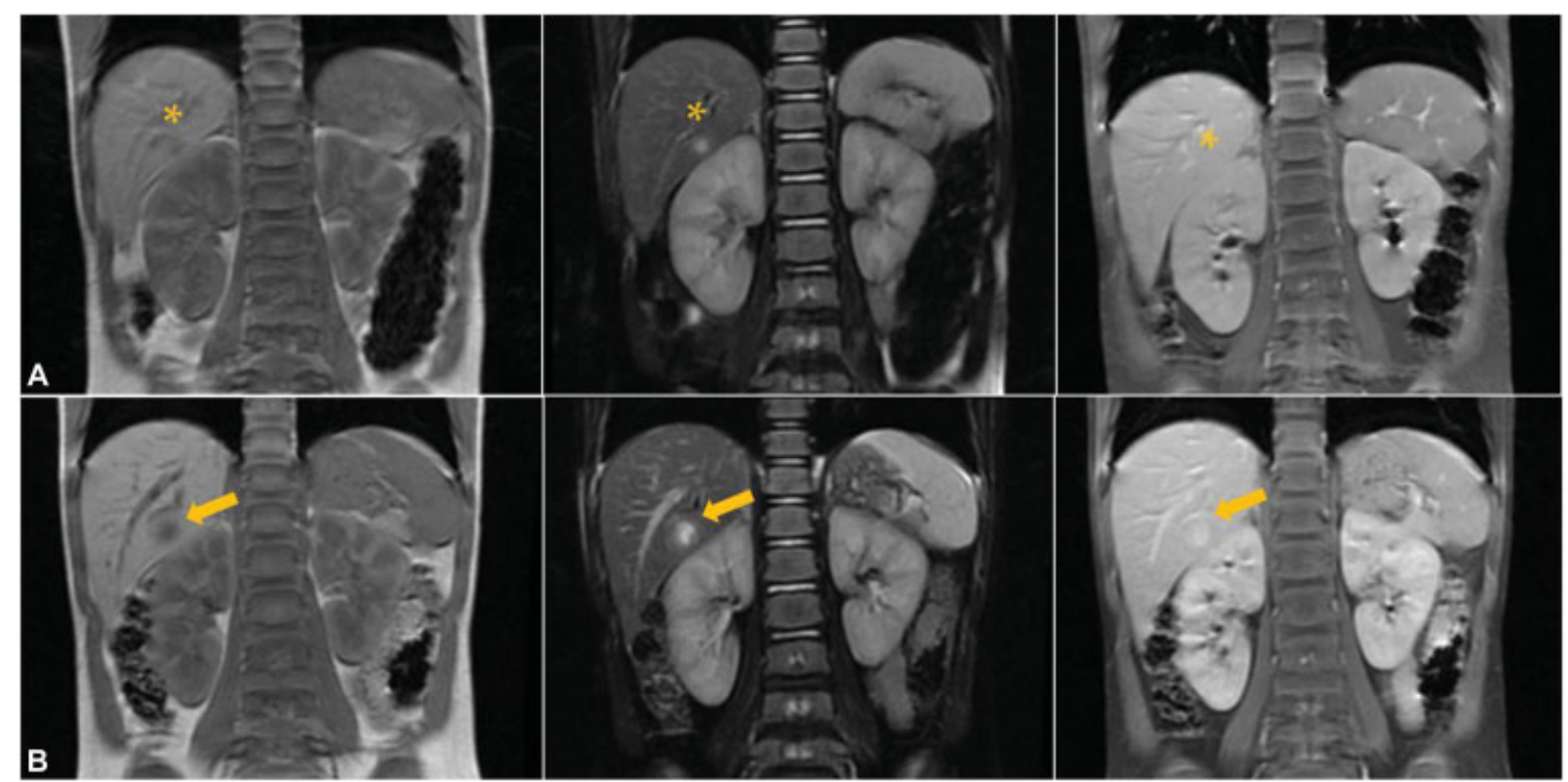

Fig. 3 Magnetic resonance imaging of the abdomen coronal T1, T2, and T1 + c images: 3 (A) and 6 (B) months follow-up, no enhancing soft tissue at the surgical bed (left suprarenal region), to suggest tumor recurrence. Lesion within segment $6 / 5$ shows interval increase in size with definite enhancement on postcontrast image; likely metastases, biopsy was recommended.

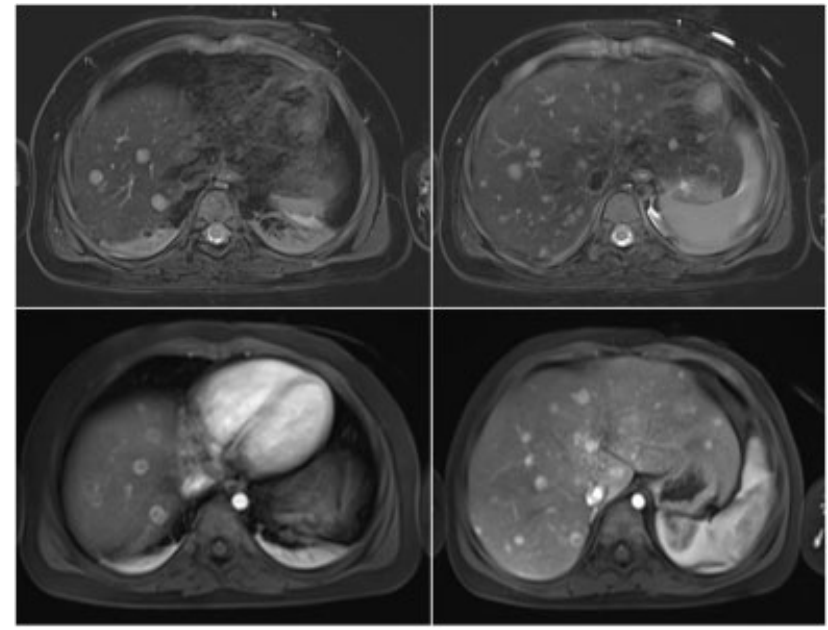

Fig. 4 Magnetic resonance imaging of the abdomen axial $T 2$ and $T 1+c$ images: Show interval increase in size and number of lesions scattered in liver parenchyma 3 months later after additional chemotherapy cycles.

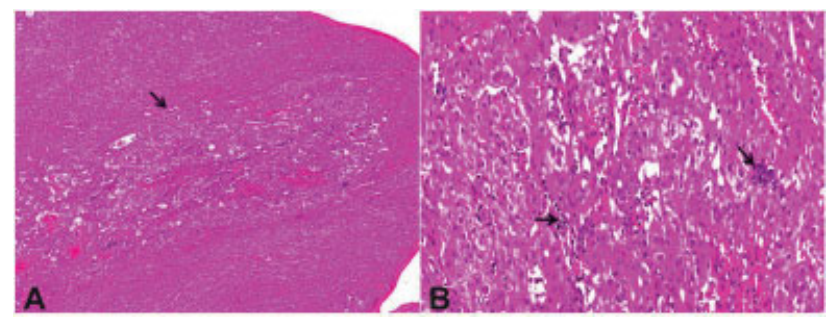

Fig. 5 Postbiopsy: (A) Low-power (HPS $\times 40$ ) liver parenchyma showing a vague nodule (arrow marking nodule of extramedullary hematopoiesis). (B) Medium-power (HPS $\times 200$ ) views of the liver parenchyma showing dilated sinusoids, extramedullary hematopoiesis, and sinusoidal capillarization.

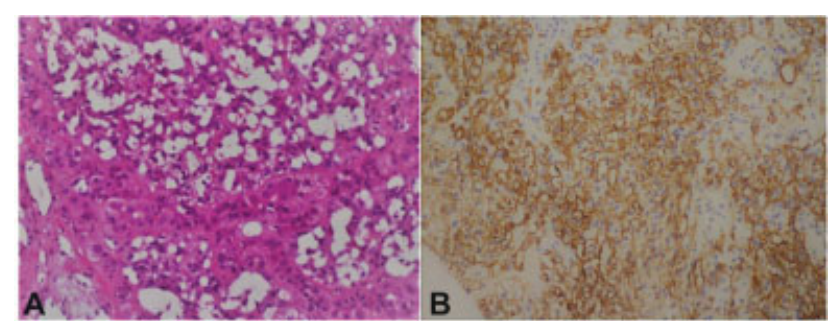

Fig. 6 Postbiopsy: (A) High-power liver parenchyma and (B) CD 34 immunostain showing pseudometastatic hypervascular lesion with extensive parenchymal loss and sinusoidal capillarization.

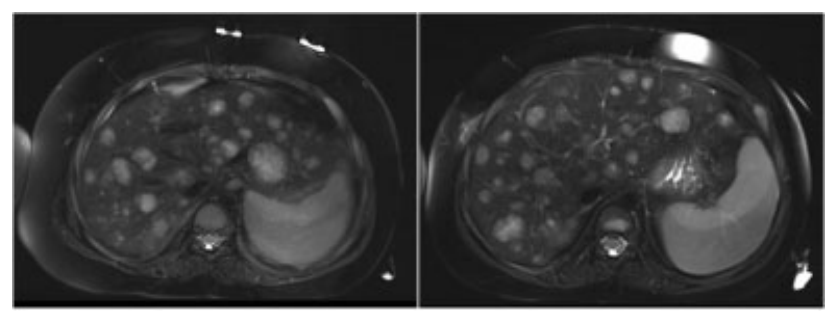

Fig. 7 Magnetic resonance imaging of the abdomen axial T2 images: Show stable large liver lesions 6 years after presentation. These lesions were found to be a combination of extramedullary hematopoiesis and pseudometastatic hypervascular lesions.

development of multiple liver lesions. At pathology, they were confirmed to be a combination of EMH and pseudometastatic hypervascular lesions.

\section{Discussion}

Neuroblastoma is a common neoplasm in pediatric age group, usually presenting between 1 and 5 years of age. Metastases are frequent in bones and lymph nodes. 
Occasionally, they may also be seen within the visceral organs and soft tissues. Therapeutic strategy strongly depends on initial staging with multimodality imaging. With improving outcomes, these patients are under follow-up for extensive periods. The case report highlights imaging findings that may present months and years after initial treatment.

EMH is production of normal blood cells outside of the bone marrow. It is a compensatory mechanism which is seen when there is inadequate functioning of medullary hematopoiesis. $^{2}$ The pathogenesis of EMH in patients with solid tumors remains unclear. Granulocyte colony-stimulating factor (G-CSF) may be an inducing factor of EMH. Our patient had received G-CSF due to chemotherapy-related bone marrow suppression. As a growth factor, G-CSF can stimulate the bone marrow to produce granulocytes, thereby increasing the release of granulocytes into the blood. ${ }^{3}$ This may suggest that in these cases, EMH may represent a reactive condition due to an increase in demand for blood elements. Additionally, chemotherapeutic agents may also play roles in the pathogenesis of EMH.

A recent review described the presence of $\mathrm{EMH}$ in a spectrum of malignant solid tumors. They also describe liver as the most common visceral organ for EMH followed by kidney and paraspinal region. ${ }^{3}$ However, the association of neuroblastoma and EMH in liver has not been reported in the literature.

Pseudometastatic hypervascular mass lesions are thought to belong to the spectrum of focal nodular hyperplasia (FNH) or regenerative nodular hyperplasia $(\mathrm{RNH}){ }^{5,6}$ In general, FNH is an uncommon lesion in the pediatric population and is usually found incidentally as a solitary mass in an otherwise normal liver. ${ }^{7}$ On the other hand, $\mathrm{RNH}$, also a rare finding in pediatric population, is characterized by nodules surrounded by atrophic liver in the absence of fibrosis.

In an initial work in the spectrum of nodules found in childhood survivors of cancer, Brisse et $\mathrm{al}^{8}$ in a retrospective multicenter series described hypervascular nodules developing 15 months to 16 years after treatment. Studies by Lee et $\mathrm{al}^{5}$ and Yoo et $\mathrm{al}^{6}$ investigated imaging findings and clinical features of benign hypervascular hepatic nodules in childhood-cancer survivors. The majority of the patients in their study had neuroblastoma. It was suggested that the nodules developing in these children showed an overlap of radiological and pathological features which could represent either RNH and/or FNH. A specific study in a subset of long-term survivors of neuroblastoma by Benz-Bohm et $\mathrm{al}^{9}$ suggested that nodules developing in cancer survivors are likely to be FNH. Some, but not all of these lesions were confirmed on histopathology. Surprisingly, in our case, the pathological features were not specific for either FNH or RNH.

Multiple previous studies have described chemotherapy with/without radiotherapy and stem cell transplant may also play a pertinent role in the development of these hypervascular lesions specifically in cases of children with neuroblastoma. ${ }^{9}$ Although the exact pathogenesis remains unclear, this phenomenon has been attributed to vascular injuries by antineoplastic therapy leading to thrombosis with pressure changes and eventual nodular hyperplasia. It has been previously speculated that particular types of chemotherapy agents such as cyclophosphamide, busulfan, and melphalan can attribute to development of FNH and $\mathrm{RNH}$. These are cytoreductive agents administered before bone marrow transplantation and have been incriminated in hepatic veno-occlusive disease. ${ }^{10}$ This brings us back to the possible proposed pathogenesis eventually leading to development of hypervascular nodules. Chemotherapy protocol in our case included doxorubicin, cyclophosphamide, and etoposide.

In summary, in our case of neuroblastoma, multiple hypervascular liver lesions were detected which were not characteristic for either FNH or RNH on imaging. Our pathological assessment was corroborative of a unique subset of hypervascular lesions, not typical for either FNH and/or RNH. Also, our case had additional nodules of EMH. In the setting of neuroblastoma, this is a finding which has not been previously described.

\section{Conclusion}

To conclude, in the setting of an existing malignancy, specifically neuroblastoma, EMH may pose as metastatic deposits signifying progression of disease. Additionally, childhood cancer survivors are at risk of developing pseudometastatic hypervascular mass lesions, which are often multiple and develop years after completing chemotherapy. Due to prognostic implications, it is essential to confirm these lesions as they are nonaggressive with a benign course.

\section{Declaration of Patient Consent}

The authors certify that they have obtained all appropriate patient consent forms. In the form, the patient's guardian has given her consent for her images and other clinical information to be reported in the journal. The patient's guardian understands that her name and initials will not be published and due efforts will be made to conceal her identity, but anonymity cannot be guaranteed.

\section{Financial Support and Sponsorship}

Nil.

\section{Conflict of Interest}

None declared.

\section{Acknowledgments}

This work presented with Caffey Award for best case report poster at the SPR 2019, Society of Pediatric Radiology Annual Meeting and Postgraduate Course at San Francisco.

\section{References}

1 Swift CC, Eklund MJ, Kraveka JM, Alazraki AL. Updates in diagnosis, management, and treatment of neuroblastoma. Radiographics 2018;38(02):566-580 
$2 \mathrm{Kim} \mathrm{CH}$. Homeostatic and pathogenic extramedullary hematopoiesis. J Blood Med 2010;1:13-19

3 Bao Y, Liu Z, Guo M, Li B, Sun X, Wang L. Extramedullary hematopoiesis secondary to malignant solid tumors: a case report and literature review. Cancer Manag Res 2018;10:1461-1470

4 Georgiades CS, Neyman EG, Francis IR, Sneider MB, Fishman EK. Typical and atypical presentations of extramedullary hemopoiesis. AJR Am J Roentgenol 2002;179(05):1239-1243

5 Lee MH, Yoo S-Y, Kim JH, et al. Hypervascular hepatic nodules in childhood cancer survivors: clinical and imaging features. Clin Imaging 2012;36(04):301-307

6 Yoo SY, Kim JH, Eo H, Jeon TY, Sung KW, Kim HS. Dynamic MRI findings and clinical features of benign hypervascular hepatic nodules in childhood-cancer survivors. AJR Am J Roentgenol 2013;201(01):178-184
7 Chung EM, Lattin GE Jr, Cube R, et al. From the archives of the AFIP: Pediatric liver masses: radiologic-pathologic correlation. Part 2. Malignant tumors. Radiographics 2011;31(02): 483-507

8 Brisse H, Servois V, Bouche B, et al. Hepatic regenerating nodules: a mimic of recurrent cancer in children. Pediatr Radiol 2000;30 (06):386-393

9 Benz-Bohm G, Hero B, Gossmann A, Simon T, Körber F, Berthold F. Focal nodular hyperplasia of the liver in longterm survivors of neuroblastoma: how much diagnostic imaging is necessary? Eur J Radiol 2010;74(03):e1-e5

10 Coppell JA, Richardson PG, Soiffer R, et al. Hepatic veno-occlusive disease following stem cell transplantation: incidence, clinical course, and outcome. Biol Blood Marrow Transplant 2010;16(02): 157-168 